\title{
Mark Abel, Groove. An aesthetic of measured time
}

Chicago, Haymarket books, 2015

\section{Frederico Lyra de Carvalho}

\section{(2) OpenEdition}

\section{Journals}

Édition électronique

URL : http://journals.openedition.org/transposition/5737

DOI : 10.4000/transposition. 5737

ISSN : 2110-6134

Éditeur

CRAL - Centre de recherche sur les arts et le langage

Référence électronique

Frederico Lyra de Carvalho, «Mark Abel, Groove. An aesthetic of measured time », Transposition [En ligne], 9 | 2021, mis en ligne le 15 décembre 2020, consulté le 23 avril 2021. URL : http://

journals.openedition.org/transposition/5737 ; DOI : https://doi.org/10.4000/transposition.5737

Ce document a été généré automatiquement le 23 avril 2021.

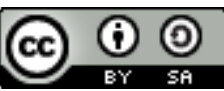

La revue Transposition est mise à disposition selon les termes de la Licence Creative Commons Attribution - Partage dans les Mêmes Conditions 4.0 International. 


\title{
Mark Abel, Groove. An aesthetic of measured time
}

\author{
Chicago, Haymarket books, 2015
}

Frederico Lyra de Carvalho

\section{RÉFÉRENCE}

Mark Abel, Groove. An aesthetic of measured time, Chicago, Haymarket books, 2015, 275 p.

Dans son ouvrage Groove. An aesthetic of measured time, Mark Abel se propose de définir le phénomène du groove comme une expression musicale et sociale propre au $\mathrm{xx}^{\mathrm{e}}$ siècle. L'intention de l'auteur est de partir du concept de groove pour faire une critique de la temporalité du système capitaliste. Venant d'une tradition marxiste, l'auteur ne dissocie pas le développement qui a connu la musique populaire tout au long du siècle de celui du système socio-économique lui-même. De sorte qu'un phénomène proprement musical se trouve ainsi employé comme un outil théorique d'une critique qui va bien au-delà de l'aspect musical, sans pour autant le laisser de côté. En outre, Abel nous dit que son intérêt pour l'objet vient d'une pratique de musicien, ce qui donne un contenu d'expérience empirique à l'ouvrage qui est tout à fait remarquable. En effet, c'est un livre qui réalise un entrecroisement entre plusieurs disciplines en une forme d'histoire sociale du groove. Néanmoins, comme nous tenterons de le montrer, le choix inhabituel d'un tel objet impose une certaine difficulté. D'une part, l'auteur a le mérite de trouver dans le groove musical un noyau critique qui dépasse la musique. D'autre part, en raison de son caractère contre-intuitif, l'objet est rapidement positivé et pris comme acquis. On aurait pu attendre une critique plus nette de l'objet lui-même. Un autre problème est le fait de manquer, dans le texte, d'une définition plus précise de ce que l'auteur entend par « musique populaire».

2 Le livre se divise en sept chapitres. Dans le premier nous trouvons une définition formelle du groove. Le deuxième montre que, contrairement au sens commun, le phénomène du groove n'aurait pas de racines africaines, il serait plutôt un phénomène 
occidental. Cette thèse est primordiale pour l'argument avancé par Abel. L'auteur fait ensuite une critique de l'insuffisance de la phénoménologie pour penser l'objet groove. Selon lui, il manquerait la matérialité nécessaire pour penser ce phénomène dans ce courant philosophique. Le cinquième chapitre est dédié à une analyse de la pensée de la réification du temps chez Adorno. L'auteur marxiste qui a le plus traité de la musique occupe une place importante dans l'ouvrage; Abel suit le philosophe allemand notamment dans l'idée que les œuvres d'art doivent être comprises d'un point de vue historique, leurs contenus et formes étant des sédiments de leurs contextes. En ce sens, il faudrait inclure aussi bien Schoenberg que la musique populaire comme manifestations musicales propres au modernisme au Xxe siècle. En outre, Abel observe que, paradoxalement, en raison de son profil très critique envers les musiques populaires, Adorno les aurait bien compris d'un point de vue conceptuel et musicologique. Celui-ci aurait bien décrit ces éléments constitutifs, par exemple la syncope. Enfin, le sixième chapitre s'appuie sur plusieurs auteurs pour penser le temps dans la société capitaliste, et le septième et dernier met en rapport le temps musical avec le temps dans cette société.

3 L'auteur prend comme point de départ l'affirmation que la musique est aussi une esthétisation du temps de la société. Ainsi comme Adorno, Abel pense que la musique transforme et reflète dialectiquement le temps social et vice-versa. Son hypothèse est qu'une étude de cette relation pourrait éclairer les deux notions. La musique serait ainsi en même temps autonome et en étroite liaison avec la société à laquelle elle appartient et « l'étude du temps musical deviendrait un mécanisme qui peut éclairer la conception implicite du temps qui prédomine dans une société ${ }^{1} »($ p. 3). La musique serait donc une manière d'entendre la temporalité d'une société quelconque. Cela s'exprime dans sa forme, mais aussi dans son contenu rythmique et, dans certaines cultures, dans la métrique. Le groove serait ainsi une manière particulière d'organiser le rythme et la métrique musicale de la musique occidentale. À la fin, «il constitue quelque chose comme un changement de paradigme dans la temporalité musicale et, pour le meilleur ou pour le pire, a eu en un peu plus d'un siècle un impact considérable sur notre culture musicale et sur ce que nous comprenons comme étant la musique » (p. 1).

4 Abel dit que «le groove est un phénomène musical propre à la musique populaire occidentale du $\mathrm{xx}^{\mathrm{e}}$ siècle » (p. 24). C'est un processus musical qui émerge notamment dans des traditions musicales entièrement ou partiellement improvisées et se manifeste normalement sur des structures répétitives de mesures en 4/4. Le groove consiste, selon Abel, en une "constellation» (p. 24) de quatre éléments qui doivent toujours être présents: (1) Le temps métronomique. Autrement dit, la musique doit exprimer une adhésion régulière à une pulsation isochrone. Il est donc nécessaire d'entendre d'une manière ou d'une autre la pulsation. L'auteur n'oublie pas de nous rappeler que ce fait n'est pas transhistorique, car nous ne trouvons pas cette régularité dans certaines musiques anciennes ni chez d'autres cultures. (2) La syncope. Il définit ce phénomène comme : « le désalignement délibéré des notes soulignées dans une partie musicale par rapport à la pulsation sous-jacente de la musique » (p. 32). La syncope s'exprime en contradiction avec la pulsation, créant ainsi un effet d'anticipation ou de retard dans l'écoute. (3) Profondeur métrique (ou à plusieurs niveaux). C'est la manière d'organiser formellement le groove avec le partage des différents rythmes entre les divers instruments impliqués. Il est très important que chaque partie soit continuellement 
accentuée. La combinaison entre les instruments et les accents est réitérée durant le morceau et c'est cette répétition qui crée une structure métrique. (4) Contretemps (back-beat). À la différence d'autres musiques, dans la musique groove, nous avons une emphase sur le deuxième et le quatrième temps de la mesure $4 / 4$.

L'approche du groove comme constellation est intéressante, car elle explicite bien la nécessité d'avoir les quatre paramètres pour pouvoir créer du groove. Mais elle pose aussi des problèmes. D'une part, un élément présuppose l'autre. Par exemple, l'apparition de la syncope présuppose un temps isochrone. D'autre part, les éléments finissent par se superposer. C'est le cas notamment de la syncope qui donne elle aussi de la profondeur au morceau. Un autre problème est que l'auteur nous donne peu d'exemples musicaux et qu'ils appartiennent tous à une même période, qui s'étend des années 1960 aux années 1980. Il aurait pu les étendre à l'ensemble du Xxe siècle pour ainsi mieux historiciser sa théorie et son importance tout au long de cette période.

6 L'auteur nous rappelle qu'« il ne faut pas se méprendre sur le fait que la plupart des musiques d'aujourd'hui dites folkloriques sont interprétées selon les conventions contemporaines et ont donc été transformées en musique groove» (p. 59). En tant que phénomène occidental, le devenir groove des toutes les musiques du monde fait aussi partie de l'expansion de la domination du système capitaliste à travers le marché global. Ce qui l'amène à soutenir que, contrairement au lieu commun, le groove n'est pas d'origine africaine. Selon lui, cet argument serait anhistorique et essentialiste. Il observe que même dans le blues nord-américain, nous pouvons trouver des éléments d'origine européenne, voire asiatique. S'appuyant sur Gerhard Kubik et Simha Arom, Abel démontre que, malgré le fait d'avoir une régularité rythmique, les musiques africaines ne sont pas métriques. Comme nous ne trouvons ni d'accentuation régulière ni non plus de syncopes, elles seraient cycliques plutôt que métriques. De sorte que nous ne trouvons pas dans les musiques africaines les quatre paramètres constituants du groove. D'autre part, la proximité des musiciens afro-américains avec le continent africain durant le $\mathrm{xx}^{\mathrm{e}}$ siècle aurait ainsi été beaucoup plus liée à leurs choix esthétiques et politiques. C'est pour cette raison que la musique nord-américaine est devenue au cours du siècle, pour ainsi dire, de plus en plus noire.

7 La métrique est un principe organisationnel temporel qui gouverne pratiquement tous les aspects de la vie. La distinction faite par Moishe Postone entre, d'un côté, un temps concret et non-linéaire et structurant ${ }^{2}$ et d'autre côté un temps abstrait et mathématisé, capable d'accueillir pratiquement n'importe quel contenu est reprise par Abel. Ce temps abstrait s'étend à partir de la Renaissance en Occident non seulement par des moyens technologiques, comme la diffusion des horloges, mais aussi pour des raisons sociales. Cette extension est possible notamment à cause de la centralité croissante que le travail acquiert dans la société capitaliste naissante. Nous trouvons une mise en discipline du temps qui se voit ainsi défini de l'extérieur, subsumé à une mesure préalable. C'est aussi le cas pour la musique (p. 187-221).

8 L'apparition du groove au xxe siècle fait partie de l'histoire de la rationalisation de la métrique dans la musique en occident. L'auteur nous rappelle d'abord que du chant grégorien jusqu'au début de la Renaissance, la musique n'était pas sous l'emprise de la métrique et que le processus de métrification va de pair avec le développement de l'écriture. En deux mots: «la mesure musicale émerge dans certaines conditions historiquement déterminées, conditions qui se sont développées d'abord en Europe et qui sont souvent regroupées sous le terme générique de "modernité", mais qui 
devraient peut-être être identifiées plus spécifiquement comme associées à la montée du capitalisme, ou au moins de la "société bourgeoise" »(p. 89). La métrique serait donc un phénomène propre à la musique créée dans la société capitaliste qui s'est répandu dans la musique africaine, asiatique et latino-américaine à travers la globalisation de l'industrie culturelle durant le $\mathrm{xx}^{\mathrm{e}}$ siècle. En suivant le fil, le groove pourrait paradoxalement être perçu comme étant en même temps un phénomène esthétique et de domination culturelle. C'est ainsi que, pour mieux lever cette ambiguïté résultant de l'argumentaire de l'ouvrage, il conviendrait d'apporter une critique plus explicite au phénomène du groove en lui-même. De sorte que, sans cette critique, il nous semble difficile de nous accorder avec Mark Abel au moment où, dans sa conclusion, il soutient que le groove serait « une pratique esthétique qui, à travers la saisie mimétique du temps mesuré du capitalisme, réalise la figuration de la praxis d'une temporalité libérée.» (p. 256).

\section{NOTES}

1. Toutes les traductions sont de notre responsabilité.

2. Par exemple : le temps scatologique.

\section{AUTEURS}

\section{FREDERICO LYRA DE CARVALHO}

Frederico Lyra de Carvalho est musicien et philosophe. Il est doctorant à l'Université de Lille en philosophie de l'art sur la direction de la professeure Anne Boissière. Sa thèse s'intitule :

Dialectique négative, improvisation, jazz : à partir de la musique. Il a publié plusieurs travaux concernant la pensée d'Adorno, l'École de Francfort, Paulo Arantes, la critique de l'écoute et la musique brésilienne. Il fait partir du comité d'édition des revues Sinal de Menos et Passages de Paris. 